Pacific Journal of Mathematics

ON EXTENDING HIGHER DERIVATIONS GENERATED BY
CUP PRODUCTS TO THE INTEGRAL CLOSURE 


\title{
ON EXTENDING HIGHER DERIVATIONS GENERATED BY CUP PRODUCTS TO THE INTEGRAL CLOSURE
}

\author{
Joseph Becker and William C. Brown
}

Let $A=k\left[x_{1}, \cdots, x_{g}\right]$ be a finitely generated integral domain over a field $k$ of characteristic zero. Let $\bar{A}$ denote the integral closure of $A$ in its quotient field. A well known result due to A. Seidenberg says that any first order $k$-derivation of $A$ can be extended to $\bar{A}$. This result is known to be false for higher order derivations. In this paper, the authors investigate what types of higher derivations on $A$ can be extended to $\bar{A}$. The main results are for higher derivations which are cup products. Set $\operatorname{Der}_{k}^{1}(A)=\operatorname{Der}_{k}^{1}(A)_{0}$ and inductively define $\operatorname{Der}_{k}^{n}(A)_{0}$ as follows:

$$
\operatorname{Der}_{k}^{n}(A)_{0}=\left\{\varphi \in \operatorname{Der}_{k}^{n}(A) \mid \Delta \varphi \in \sum_{i=1}^{n-1} \operatorname{Der}_{k}^{2}(A)_{0} \cup \operatorname{Der}_{k}^{n-i}(A)_{0}\right\}
$$

The authors show that if $\varphi \in \operatorname{Der}_{k}^{n}(A)_{0}$, then $\varphi(\bar{A}) \subseteq \bar{A}$. Various examples are given which indicate that the above mentioned result is about as good as possible.

Introduction. Throughout this paper, $A=k\left[x_{1}, \cdots, x_{g}\right]$ will denote a finitely generated integral domain over a field $k$ of characteristic zero. We shall let $Q$ denote the quotient field of $A$ and $\bar{A}$ the integral closure of $A$ in $Q$. For each $n=1,2, \cdots$, we shall let $\operatorname{Der}_{k}^{n}(A)$ denote the $A$-module of all $n$th order $k$-derivations of $A$ to $A$. Thus, $\varphi \in \operatorname{Der}_{k}^{n}(A)$ if and only if $\varphi \in \operatorname{Hom}_{k}(A, A)$, and for all $a_{0}, \cdots, a_{\imath} \in A$ we have

(1) $\varphi\left(a_{0} a_{1} \cdots a_{n}\right)=\sum_{s=1}^{n}(-1)^{s-1} \sum_{i_{1}<\cdots<i_{s}} a_{i_{1}} \cdots a_{i_{s}} \varphi\left(a_{0} \cdots \check{a}_{i_{1}} \cdots \check{a}_{i_{s}} \cdots a_{n}\right)$.

The authors refer the reader to [3] for the various facts about $\operatorname{Der}_{k}^{n}(A)$ used in this paper. Of particular importance is the fact that any $n$th order derivation $\varphi \in \operatorname{Der}_{k}^{n}(A)$ can naturally be extended to an $n$th order derivation of any localization of $A$ [Thm 15;3].

We shall need the Hochschild coboundary operator $\Delta$ which is defined as follows: If $\varphi \in \operatorname{Hom}_{k}(A, A)$, then $\Delta \varphi: A \times A \rightarrow A$ is the $k$-bilinear mapping defined by $\Delta \varphi\left(a_{1}, a_{2}\right)=\varphi\left(a_{1} a_{2}\right)-a_{1} \varphi\left(a_{2}\right)-a_{2} \varphi\left(a_{1}\right)$. We shall also need the cup product $\varphi \cup \psi$ of two $k$-linear mappings $\varphi$ and $\psi$ of $A$. $\varphi \cup \psi: A \times A \rightarrow A$ is the $k$-bilinear mapping defined by $\varphi \cup \psi\left(a_{1}, a_{2}\right)=\varphi\left(a_{1}\right) \psi\left(a_{2}\right)$ If $P$ and $P$ are two $A$-submodules of $\operatorname{Hom}_{k}(A, A)$, then $P \cup P$ will denote the set of all $k$-bilinear mappings of $A \times A$ into $A$ which are finite $A$-linear combinations of mappings 
of the form $\varphi \cup \psi$ for $\varphi \in P, \psi \in P^{\prime}$. Thus, if $\varphi$ is an $n$th order $k$ derivation of $A$ such that $\Delta \varphi \in \sum_{i=1}^{n-1} \operatorname{Der}_{k}^{i}(A) \cup \operatorname{Der}_{k}^{n-i}(A)$, then there exist constants $e_{l j} \in A$ and $k$-derivations $\psi_{l}^{(j)}, \lambda_{l}^{(j)} \in \operatorname{Der}_{k}^{j}(A)$ such that for all $a$ and $b$ in $A$, we have

$$
\begin{aligned}
\varphi(a b)= & a \varphi(b)+b \varphi(a)+\sum e_{l 1} \psi_{l}^{(1)}(a) \lambda_{l}^{(n-1)}(b)+\cdots \\
& +\sum e_{l n-1} \psi_{l}^{(n-1)}(a) \lambda_{l}^{(1)}(b) .
\end{aligned}
$$

Now the purpose of this paper is to study which $n$th order $k$ derivations $\varphi: A \rightarrow A$ can be extended to $\bar{A}$. In [4], A. Seidenberg showed that any 1st order derivation of $A$ must map $\bar{A}$ to $\bar{A}$. In [1], an example was given which shows that 2nd order derivations $\varphi \in \operatorname{Der}_{k}^{2}(A)$ need not have the property that $\varphi(\bar{A}) \subset \bar{A}$. Since we shall have use of this example latter, we present it here

EXAMPLE 1. Consider the curve $X^{2}=Y^{3}$ over the rational numbers $\boldsymbol{Q}$. Let $A$ be the coordinate ring of this curve i.e. $A=Q[x, y]=$ $\boldsymbol{Q}[X, Y] /\left(X^{2}-Y^{3}\right)$. One can easily check that $A$ is a domain whose integral closure is given by $\bar{A}=A[x / y]$. Since the quotient field of $A$ is a finite separable extension of $\boldsymbol{Q}(y)$, it follows that any 2 nd order derivation $\varphi \in \operatorname{Der}_{Q}^{2}(A)$ is determined by its values on $y$ and $y^{2}$. A simple calculation shows that if $\varphi(y)=a$, and $\varphi\left(y^{2}\right)=b$ (where $a$ and $b$ lie in the quotient field of $A$ ), then

$$
\varphi(x)=\frac{3 y}{8}\left(\frac{2 y a+b}{x}\right), \varphi\left(x^{2}\right)=3 y b-3 y^{2} a
$$

and

$$
\varphi(x y)=\frac{5 y^{2}}{8}\left(\frac{3 b-2 y a}{x}\right) .
$$

If we set $a=1$ and $b=-2 y$, then $\varphi \in \operatorname{Der}_{Q}^{2}(A)$, and one easily checks that $\varphi(x / y)=x / y^{2} \notin \bar{A}$.

Thus, higher derivations on $A$ need not extend to $\bar{A}$. At the end of [1], the author conjectured that any $\varphi \in \operatorname{Der}_{k}^{2}(A)$ such that $\Delta \varphi \in \operatorname{Der}_{k}^{1}(A) \cup \operatorname{Der}_{k}^{1}(A)$ must map $\bar{A}$ to $\bar{A}$. In this paper, we shall show that this conjecture is correct. We shall also formulate sufficient conditions on $\varphi \in \operatorname{Der}_{k}^{n}(A)$ in order that $\varphi(\bar{A}) \subset \bar{A}$. We assume the reader is familiar with [1].

Main results.

THEOREM 1. Let $A=k\left[x_{1}, \cdots, x_{g}\right]$ be a finitely generated integral domain over a field $k$ of characteristic zero. Let $\bar{A}$ denote the integral closure of $A$ in its quotient field $Q$. Let $\phi \in \operatorname{Der}_{k}^{2}(A)$ and 
assume $\Delta \varphi \in \operatorname{Der}_{K}^{1}(A) \cup \operatorname{Der}_{k}^{1}(A) . \quad$ Then $\varphi(\bar{A}) \subset \bar{A}$.

Proof. Let $\operatorname{Min}(\bar{A})$ denote the collection of height one primes in $\bar{A}$. Since $\bar{A}$ is a Krull domain, we have $\bar{A}=\bigcap\left\{\bar{A}_{q} \mid q \in \operatorname{Min}(\bar{A})\right\}$. Here as usual $\bar{A}_{q}$ means $\bar{A}$ localized at the prime $q$. Let $q \in \operatorname{Min}(\bar{A})$. Then $p=q \cap A \in \operatorname{Min}(A)$. Let us set $R=A_{p}$ and $\bar{R}=(\bar{A})_{p}=\bar{A}_{p}$ the integral closure of $R$ in $Q$. Let $\bar{q}$ denote the extended prime ideal $q \bar{R}$ in $\bar{R}$. Then $\bar{R}_{\bar{q}}=\bar{A}_{q}$. Now since $R$ is a localization of $A$, we see that $\varphi \in \operatorname{Der}_{k}^{2}(R)$. Suppose we could show that $\varphi(\bar{R}) \subseteq$ $\bar{R}$. Then $\varphi\left(\bar{R}_{\bar{q}}\right) \subseteq \bar{R}_{\bar{q}}$ or equivalently $\varphi\left(\bar{A}_{q}\right) \subseteq \bar{A}_{q}$. Since $\bar{A}$ is the intersection of the $\bar{A}_{q}$, the theorem would be proven. Thus to prove Theorem 1, it suffices to prove the following assertion:

"Under the same hypotheses as Theorem 1, let $p \in \operatorname{Min}(A), R=$ $A_{p}$ and $\bar{R}=\bar{A}_{p}$. Then $\varphi(\bar{R}) \subseteq \bar{R}$."

So fix a minimal prime $p \in \operatorname{Min}(A)$, and set $R=A_{p}, \bar{R}=\bar{A}_{p}$. We have already noted that $\varphi \in \operatorname{Der}_{k}^{2}(R)$, and one easily sees that $\Delta \varphi \in$ $\operatorname{Der}_{k}^{1}(R) \cup \operatorname{Der}_{k}^{1}(R)$. Now if $A=\bar{A}$, there is nothing to prove. Hence, we may assume $\bar{A} \neq A$. Then the conductor $C$ of $A$ in $\bar{A}$ is a proper ideal in $A$. If $C \not \subset p$, then $R=\bar{R}$ and again there is nothing to prove. Hence we may assume $C \subset p$. In this case, $C R$ is the conductor of $R$ in $\bar{R}$.

We now follow the proof of Theorem 3 in [1]. Let the transcendence degree of $A$ over $k$ be $r$, and let $m$ denote the maximal ideal in $R$. Then $R / m$ is the quotient field of $A / p$ and hence has transcendence degree $r-1$ over $k$. Let $\left\{\bar{a}_{1}, \cdots, \bar{a}_{r-1}\right\}$ be a transcendence basis of $R / m$ over $k$. Pull these $\bar{a}_{i}$ back to elements $\alpha_{i}$ in $R-$ $m$. Then $F=k\left(\alpha_{1}, \cdots, \alpha_{r-1}\right)$ is a field of transcendence degree $r-1$ over $k$, and $F \subset R$.

We know that $\bar{R}$ is a semilocal ring with maximal ideals $m_{1}$, $\cdots, m_{t}$ lying over $m$ in $R$. Set $J=\bigcap_{i=1}^{t} m_{i}$, the Jacobson radical of $\bar{R}$. Each local ring $V_{i}=\bar{R}_{m_{i}}, i=1, \cdots, t$, is a discrete rank one valuation ring dominating $R$. By [Thm 18, p. 45; 6], we can find an element $\beta \in J$ such that $\beta$ generates the maximal ideal in each $V_{i}$. Since the Krull dimension of $\bar{R}$ is one, we see that $J$ is the radical of the ideal $C R$ in $\bar{R}$. Thus, some power of $\beta$, say $\beta^{n}$, lies in $C R$. We shall have use of this remark later.

It was shown in [1], that $\operatorname{Der}_{k}^{1}(\bar{R})$ is a free $\bar{R}$-module with basis $\left\{\delta_{0}, \delta_{1}, \cdots, \delta_{r-1}\right\}$. The derivations $\delta_{i}$ satisfy the following relations:

$$
\delta_{0}(\beta)=1, \delta_{0}\left(\alpha_{i}\right)=0=\delta_{i}(\beta) \text { for } i=1, \cdots, r-1
$$




$$
\delta_{i}\left(\alpha_{j}\right)=\left\{\begin{array}{ll}
1 & \text { if } \quad i=j \\
0 & \text { if } \quad i \neq j
\end{array} \quad 1 \leqq i \leqq j \leqq r-1\right.
$$

We observe that the derivations $\delta_{i}$ commute on the field $F(\beta)$. Since $\beta$ is a uniformizing parameter for $V_{i}, \beta$ is transcendental over $F$. Hence $Q$ is a separable algebraic extension of $F(\beta)$. Therefore the derivations on $F(\beta)$ have a unique extension to $Q$. It follows that the $\delta_{i}$ commute on $Q$. It follows from [2; Thm 16,11.2] that the union $\bigcup_{n=1}^{\infty} \operatorname{Der}_{k}^{n}(Q)$ is a free $Q$-algebra generated by $\delta_{0}, \cdots, \delta_{r-1}$. In particular, $\varphi$ can be written as a unique polynomial of degree two in $\delta_{0}, \cdots, \delta_{r-1}$. The coefficients of this polynomial lie in $Q$. Let us write $\varphi$ as follows:

$$
\varphi=\sum_{i=0}^{r-1} a_{i} \delta_{i}+\sum_{0 \leqq i<j \leqq r-1} a_{i j} \delta_{i} \delta_{j}+\sum_{i=0}^{r-1} a_{i i} \delta_{i}^{2} .
$$

Since $\Delta \varphi \in \operatorname{Der}_{k}^{1}(R) \cup \operatorname{Der}_{k}^{1}(R)$, we can write for all $a$ and $b$ in $R$ :

$$
\varphi(a b)=a \varphi(b)+b \varphi(a)+\sum_{l} e_{l} \psi_{l}(a) \lambda_{l}(b)
$$

where $e_{l} \in R$ and $\psi_{l}, \lambda_{l} \in \operatorname{Der}_{k}^{1}(R)$. One easily checks that equation (5) continues to hold for all $a$ and $b$ in $Q$. Now by [Thm 1; 4], each $\psi_{l}$ and $\lambda_{l}$ extends to $\bar{R}$. It then easily follows that $C R$ is differential under $\psi_{l}$ and $\lambda_{l}$, i.e. $\psi_{l}(C R) \subset C R$ and $\lambda_{l}(C R) \subset C R$. Thus, $C R$ remains differential under $\psi_{l}$ and $\lambda_{l}$ when considered as an ideal in $\bar{R}$. Hence, [Thm 1;5] implies that each $m_{i}$ in $\bar{R}$ is differential under $\psi_{l}$ and $\lambda_{l}$. Write each $\psi_{l}$ and $\lambda_{l}$ as a linear combination of $\delta_{0}, \delta_{1}, \ldots, \delta_{r-1}$ :

$$
\psi_{l}=\sum_{i=0}^{r-1} \mu_{l i} \delta_{i} \quad \lambda_{l}=\sum_{i=0}^{r-1} \gamma_{l i} \delta_{i} .
$$

Here the coefficients $\mu_{l i}$ and $\gamma_{l i}$ lie in $\bar{R}$. Then $\psi_{l}(J) \subset J$ and $\lambda_{l}(J) \subset J$ imply that $\mu_{l 0}$ and $\gamma_{l 0}$ lie in $J$. If we now substitute the expressions in equations (6) and (4) into equation (5) and then make various substitutions of the form $a, b=\alpha_{1}, \cdots, \alpha_{r-1}, \beta$, we see that all the coefficients, except possibly $\alpha_{0}$, appearing in (4) lie in $\bar{R}$. We further get that $a_{0 i} \in J$ for $i=1, \cdots, r-1$, and $a_{00} \in J^{2}$.

Thus, to complete the proof of the assertion $\varphi(\bar{R}) \subseteq \bar{R}$, we must show that $a_{0}$ in (4) lies in $\bar{R}$. We shall show this by arguing that $a_{0} \in V_{i}$ for every $i=1, \cdots, t$.

So fix an $i=1, \cdots, t$, and let $v_{i}: V_{i} \rightarrow Z$ be the valuation of $V_{i}$ given by $v_{i}(\beta)=1$. We wish to show that $v_{i}\left(a_{0}\right) \geqq 0$. Let us assume $v_{i}\left(a_{0}\right)<0$. We need the following lemma:

Lemma 1. There exist two elements $x$ and $y$ in $R$ such that (a) The value $N=v_{i}(x)$ of $x$ is the smallest positive value of 
any element in $R$.

(b) The value $v_{i}(y)$ of $y$ is not a multiple of $N$.

Proof. Since $R \subset V_{i}$, we have $v_{i}(z) \geqq 0$ for every element $z$ in $R$. So we can certainly find an element $x$ in $R$ which satisfies (a). As pointed out earlier, $\beta^{n} \in C R \subset R$. Thus, $\beta^{n+l} \in R$ for any nonnegative integer $l$.

Now suppose no $y \in R$ can be found satisfying (b). Then for every nonnegative integer $l$, we must have $n+l=v_{i}\left(\beta^{n+l}\right)$ is a multiple of $N$. This can only happen if $N=1$. We shall show this is impossible.

If $N=1$, then $x=\gamma \beta$ for some unit $\gamma$ in $V_{i}$. We want to consider

$$
\varphi(x)=\sum_{\imath=0}^{r-1} a_{i} \delta_{i}(x)+\sum_{0 \leqq i<j \leqq r-1} a_{i j} \delta_{i} \delta_{j}(x)+\sum_{i=0}^{r-1} a_{\imath \imath} \delta_{i}^{2}(x)
$$

which is an element of $R$. Now we have

$$
\begin{aligned}
\delta_{0}(x) & =\beta \delta_{0}(\gamma)+\gamma \\
\delta_{i}(x) & =\beta \delta_{i}(\gamma) \quad i=1, \cdots, r-1 \\
\delta_{0} \delta_{i}(x) & =\beta \delta_{0} \delta_{i}(\gamma)+\delta_{i}(\gamma) \quad i=1, \cdots, r-1 \\
\delta_{i} \delta_{j}(x) & =\beta \delta_{i} \delta_{j}(\gamma) \quad 0<i \leqq j \leqq r-1
\end{aligned}
$$

and

$$
\delta_{0}^{2}(x)=\beta \delta_{0}^{2}(\gamma)+2 \delta_{0}(\gamma) .
$$

Since the $\delta_{j}$ are derivations on $\bar{R}$, they naturally extend to $V_{i}$. Thus, the elements in equation (7) are all elements of $V_{\imath}$, and clearly $\delta_{0}(x)$ is a unit in $V_{i}$. If we now use the facts that $a_{1}, \cdots, a_{r-1}, a_{i j} \in \bar{R}$, $a_{0 i} \in J$ and $a_{00} \in J^{2}$, we see that

$$
v_{i}\left[\sum_{i=1}^{r-1} a_{i} \delta_{i}(x)+\sum_{0 \leqq i<j \leqq r-1} a_{i j} \delta_{i} \delta_{j}(x)+\sum_{i=0}^{r-1} a_{i i} \delta_{i}^{2}(x)\right] \geqq 1
$$

Thus, $v_{i}(\varphi(x))=v_{i}\left(a_{0}\right)+v_{i}\left(\delta_{0}(x)\right)=v_{\imath}\left(a_{0}\right)<0$. But, $\varphi(x) \in R$ means the value of $\varphi(x)$ must be nonnegative. Thus, we have reached a contradiction and the proof of Lemma 1 is complete.

Now among all the elements $z$ of $R$ such that $v_{i}(z)$ is not a multiple of $N$ pick one, say $y$, of smallest value $M$. Lemma 1 guarantees that such an element $y \in R$ exists. Then $M-N>0$, and $M-$ $N$ is not the value of any element of $R$. Since $v_{2}(x)=N, x=\gamma \beta^{N}$ for some unit $\gamma \in V_{i}$. An argument similar to that in Lemma 1 shows that $v_{i}(\varphi(x))=v_{i}\left(a_{0}\right)+N-1$. Now there are two cases to consider. Either $\varphi(x)$ is a unit in $R$ or it is not. If $\varphi(x)$ is a nonunit, then $v_{i}(\varphi(x)) \geqq N$. But this implies $v_{i}\left(a_{0}\right) \geqq 1$ which is contrary to 
our assumption. Thus, $\varphi(x)$ is a unit. So $v_{i}\left(a_{0}\right)=1-N$. But now a similar computation applied to $y$ gives us that $v_{i}(\varphi(y))=v_{i}\left(a_{0}\right)+$ $M-1=M-N$. Since $\varphi(y) \in R$, and $M-N$ is not the value of anything in $R$, we have reached a contradiction.

Thus, $v_{i}\left(a_{0}\right) \geqq 0$ and the proof of Theorem 1 is complete.

In our proof of Theorem 2 below, we shall need the fact that the coefficient $a_{0}$ in equation (4) actually lies in $J$. The proof of Theorem 1 shows that $a_{0} \in \bar{R}$. To see that $a_{0} \in J$, we proceed as follows: Since $\varphi(\bar{R}) \subseteq \bar{R}$, equation (5) immediately implies that $\varphi(C R) \subseteq$ $C R$. In the notation of Theorem 1 , we wish to argue that $v_{i}\left(a_{0}\right) \geqq$ 1. Suppose $v_{i}\left(a_{0}\right)=0$. Let $N$ be the minimum positive value of any element in $C R$, and let $x \in C R$ have value $N$. Then as in Lemma 1, $v_{i}(\varphi(x))=v_{i}\left(a_{0}\right)+N-1=N-1$. Since $\varphi(x) \in C R$ this is impossible. Thus $v_{2}\left(a_{0}\right) \geqq 1$.

For Theorem 2, we shall need the following definition:

Definition. Set $\operatorname{Der}_{k}^{1}(A)_{0}=\operatorname{Der}_{k}^{1}(A)$ and inductively define $\operatorname{Der}_{k}^{n}(A)_{0}$ as follows:

$$
\operatorname{Der}_{k}^{n}(A)_{0}=\left\{\rho \in \operatorname{Der}_{k}^{n}(A) \mid \Delta \varphi \in \sum_{i=1}^{n-1} \operatorname{Der}_{k}^{i}(A)_{0} \cup \operatorname{Der}_{k}^{n-i}(A)_{0}\right\} .
$$

Thus, Theorem 1 states that if $\varphi \in \operatorname{Der}_{k}^{2}(A)_{0}$, then $\varphi(\bar{A}) \subset \bar{A}$. We can now prove the general result.

THeOREM 2. Let $A=k\left[x_{1}, \cdots, x_{g}\right]$ be a finitely generated integral domain over a field $k$ of characteristic zero. Let $\bar{A}$ denote the integral closure of $A$ in its quotient field $Q$. Let $\varphi \in \operatorname{Der}_{k}^{n}(A)_{0}$. Then $\varphi(\bar{A}) \subset \bar{A}$.

Proof. The proof proceeds along the same lines as in Theorem 1. It suffices to show that for every prime $p$ of height one in $A$, $\varphi(\bar{R}) \subset \bar{R}$. Here, as in Theorem $1, \bar{R}$ denotes the integral closure of $R=A_{p}$ in $Q$. One easily checks that $\varphi \in \operatorname{Der}_{k}^{n}(R)_{0}$. We shall adopt all the notation used in Theorem 1 . Thus, $C R$ is the conductor of $R$ in $\bar{R}$.

For the purposes of this proof, let us define $\operatorname{Der}_{k}^{n}(R)_{\bar{R}}$ inductively as follows:

$$
\begin{gathered}
\operatorname{Der}_{k}^{1}(R)_{\bar{R}}=\operatorname{Der}_{k}^{1}(R) \\
\operatorname{Der}_{k}^{n}(R)_{\bar{R}}=\left\{\varphi \in \operatorname{Der}_{k}^{n}(R) \mid \Delta \varphi \in \sum_{i=1}^{n-1} \operatorname{Der}_{k}^{i}(R)_{\bar{R}} \cup \operatorname{Der}_{k}^{n-2}(R)_{\bar{R}}\right. \\
\text { and } \varphi(\bar{R}) \subset \bar{R}\} .
\end{gathered}
$$


Then we have already proven that $\operatorname{Der}_{k}^{2}(R)_{0}=\operatorname{Der}_{k}^{2}(R)_{\bar{R}}$ in Theorem 1 , and we shall show that $\operatorname{Der}_{k}^{n}(R)_{0}=\operatorname{Der}_{k}^{n}(R)_{\bar{R}}$ for all $n$.

Now we know that $\bigcup_{n} \operatorname{Der}_{k}^{n}(Q)$ is a free $Q$-algebra generated by $\delta_{0}, \cdots, \delta_{r-1}$. Thus if $\varphi \in \operatorname{Der}_{k}^{n}(R)$, then $\varphi=g\left(\delta_{0}, \cdots, \delta_{r-1}\right)$ for some polynomial $g\left(X_{0}, \cdots, X_{r-1}\right) \in Q\left[X_{0}, \cdots, X_{r-1}\right]$ of degree less than or equal to $n$. We further know this polynomial is unique. We now need the following lemma:

Lemma 2. Let $\varphi \in \operatorname{Der}_{k}^{n}(R)_{\bar{R}}$, and write $\phi=g\left(\delta_{0}, \cdots, \delta_{r-1}\right)$. Then the coefficients of any monomials of $g$ which contain $\delta_{0}^{j}(1 \leqq j \leqq n)$ lie in $J^{j}$.

Proof. We proceed by induction on $n$. The case $n=1$ was proven in Theorem 1. The case $n=2$ was proven in Theorem 1 and the remarks following Theorem 1 . Thus, we may assume Lemma 2 has been proven for all elements of $\operatorname{Der}_{k}^{m}(R)_{\bar{R}}$ with $m<n$.

Let $\phi \in \operatorname{Der}_{k}^{n}(R)_{\bar{R}}$. Then there exist constants $e_{l j} \in R$ and derivations $\psi_{i}^{(j)}, \lambda_{l}^{(j)} \in \operatorname{Der}_{k}^{j}(R)_{\bar{R}}, j=1, \cdots, n-1$, such that for all $a$ and $b$ in $Q$ equation (2) is satisfied. Our induction hypothesis applies to the derivations $\psi_{i}^{(j)}$ and $\lambda_{l}^{(j)}$. So we can write:

$$
\begin{aligned}
& \psi_{l}^{(j)}=\sum c_{t}^{l, j} \delta_{t}+\sum c_{1_{1} t_{2}}^{l, j} \delta_{t_{1}} \delta_{t_{2}}+\cdots+\sum c_{t_{1} \cdots t_{j}}^{l, j} \cdot \delta_{t_{1}} \cdots \delta_{t_{j}} \\
& \lambda_{l}^{(j)}=\sum d_{t}^{l, j} \delta_{t}+\sum d_{t_{1} t_{2}}^{l, j} \delta_{t_{1}} \delta_{t_{2}}+\cdots+\sum d_{t_{1} \cdots t_{j}}^{l, j} \delta_{t_{1}} \cdots \delta_{t_{j}} .
\end{aligned}
$$

In (10), the coefficient of any monomial in either expression which contains $\delta_{0}^{j}$ will lie in $J^{j}$. We note that since $\psi_{i}^{(j)}, \lambda_{l}^{(j)}: \bar{R} \rightarrow \bar{R}$, all the coefficients of (10) lie in $\bar{R}$.

Now write out the polynomial $g\left(\delta_{0}, \cdots, \delta_{r-1}\right)$ which gives us $\varnothing$ as follows:

$$
\varphi=\sum a_{t} \delta_{t}+\sum a_{t_{1} t_{2}} \delta_{t_{1}} \delta_{t_{2}}+\cdots+\sum a_{t_{1} \cdots t_{n}} \delta_{t_{1}} \cdots \delta_{t_{n}} .
$$

Since $\varphi(\bar{R}) \subset \bar{R}$, one easily checks that all the coefficients $a_{t}, a_{t_{1} t_{2}}, \cdots$, $a_{t_{1} \cdots t_{n}}$ of (11) lie in $\bar{R}$. We now substitute equations (10) and (11) into (2) and get:

$$
\begin{aligned}
\sum a_{t} \delta_{t} & (a b)+\sum a_{t_{1} t_{2}} \delta_{t_{1}} \delta_{t_{2}}(a b)+\cdots+\sum a_{t_{1} \cdots t_{n}} \delta_{t_{1}} \cdots t_{t_{n}}(a b) \\
= & a\left\{\sum a_{t} \delta_{t}(b)+\cdots+\sum a_{t_{1} \cdots t_{n}} \delta_{t_{1}} \cdots \delta_{t_{n}}(b)\right\} \\
& +b\left\{\sum a_{t} \delta_{t}(a)+\cdots+\sum a_{t_{1} \cdots t_{n}} \delta_{t_{1}} \cdots \delta_{t_{n}}(a)\right\} \\
& +\sum e_{l, 1}\left\{\sum \sum_{t} c_{t}^{l, 1} \delta_{t}(a)\right\}\left\{\sum_{t} d_{t}^{l, n-1} \delta_{t}(b)+\cdots\right. \\
& \left.+\sum d_{t_{1} \cdots t_{n-1}}^{l, n-1} \delta_{t_{n}} \cdots \delta_{t_{n-1}}(b)\right\}+\cdots \\
& +\sum e_{l, n-1}\left\{\sum c_{t}^{l, n-1} \delta_{t}(a)+\cdots+\sum c_{t_{1} \cdots t_{n-1}}^{l, n-1} \delta_{t_{1}} \cdots \delta_{t_{n-1}}(a)\right\} \\
& \times\left\{\sum d_{t}^{l, 1} \delta_{t}(b)\right\} .
\end{aligned}
$$


After simplifying (12) and comparing coefficients, we see that any coefficient of (11) (except possibly for $a_{0}$ ) in a monomial containing $\delta_{0}^{j}$ lies in $J^{j}$. Thus, the lemma will be complete if we show $a_{0} \in J$.

Since $\varphi(\bar{R}) \subset \bar{R}$, one easily sees using (2) that $\varphi(C R) \subset C R$. Thus, to argue $a_{0} \in J$, one can proceed exactly as in the remarks following Theorem 1. Pick an element $x \in C R$ of minimum value $N=v_{i}(x)$. If $v_{i}\left(a_{0}\right)=0$, then $v_{i}(\varphi(x))=N-1$ which is a contradiction. This completes the proof of Lemma 2.

We now proceed to prove Theorem 2 by induction on $n$. A. Seidenberg's original result [Thm; 4], and Theorem 1 give us the case $n=1$ and $n=2$. Thus, assume Theorem 2 is correct for all $m<n$, and let $\varphi \in \operatorname{Der}_{k}^{n}(R)_{0}$. We can expand $\phi$ as in equation (2) for some choice of constants $e_{l j} \in R$ and derivations $\psi_{l}^{(j)}, \lambda^{(j)} \in \operatorname{Der}_{k}^{j}(R)_{0}$. By our induction hypothesis, $\operatorname{Der}_{k}^{j}(R)_{0}=\operatorname{Der}_{k}^{j}(R)_{\bar{R}}$. So by Lemma 2 , each $\psi_{l}^{(j)}$ and $\lambda_{l}^{(j)}$ can be written as in equation (10) with the coefficients of any monomials containing $\delta_{0}^{j}$ lying in $J^{j}$. Now write $\varphi$ as in equation (11). Following the same substitutions as in Lemma 2 , we see that all the coefficients $a_{1}, \cdots, a_{r-1}, a_{t_{1} t_{2}}, \cdots, a_{t_{1} \cdots t_{n}}$ lie in $\bar{R}$. Further, the coefficients appearing in terms containing $\delta_{0}^{j}$ lie in $J^{j}$, except possibly for $a_{0}$. Thus, as in Theorem 1, we have to argue that $v_{i}\left(a_{0}\right) \geqq 0$ for all $i=1, \cdots, t$. But this argument is exactly the same as in Theorem 1. Assume $v_{i}\left(a_{0}\right)<0$. The coefficients of (11) lying in the right powers of $J$ exactly mean that $v_{i}(\varphi(z))=v_{i}\left(a_{0}\right)+$ $v_{i}(z)-1$ for any nonunit $z$ of $R$. Thus we proceed exactly as before to argue that $v_{i}\left(a_{0}\right)<0$ is impossible. This completes the proof of Theorem 2.

The reader may be wondering if a slightly weaker hypothesis on $\varphi \in \operatorname{Der}_{k}^{n}(A)$ will imply $\varphi(\bar{A}) \subset \bar{A}$. In particular, it is natural to ask the following question: Suppose $\varphi \in \operatorname{Der}_{k}^{n}(A)$ such that

$$
\Delta \varphi \in \sum_{i=1}^{n-1} \operatorname{Der}_{k}^{i}(A) \cup \operatorname{Der}_{k}^{n-i}(A) .
$$

Then is $\varphi(\bar{A}) \subseteq \bar{A}$ ? Theorem 1 implies this is true if $n=2$. We shall give an example which shows that for $n>2$ the answer to the above question is in general negative.

ExAmple 2. We return to Example 1 at the beginning of this paper. We may equally well describe the $\operatorname{ring} A$ as $A=Q\left[t^{3}, t^{2}\right]$. Set $\delta=\partial / \partial_{t}$, a first order derivation on the quotient field of $A$. One can easily check that $t \delta, t^{2} \delta, \delta^{2}-(2 / t) \delta, t \delta^{2}-\delta$ and $\delta^{3}-(3 / t) \delta^{2}+\left(3 / t^{2}\right) \delta$ are all derivations on $A$. Set 


$$
\varphi=t^{2} \delta\left(\delta^{3}-\frac{3}{t} \delta^{2}+\frac{3}{t^{2}} \delta\right)-\frac{9 t}{2} \delta\left(\delta^{2}-\frac{2}{t} \delta\right)+\frac{3}{2}\left(\delta^{2}-\frac{2}{t} \delta\right)(t \delta) .
$$

Then $\varphi \in \operatorname{Der}_{Q}^{4}(A)$. If we expand $\varphi$ out, we get $\varphi=t^{2} \delta^{4}-6 t \delta^{3}+$ $15 \delta^{2}-(18 / t) \delta$. Now the integral closure $\bar{A}$ of $A$ is just $Q[t]$, and thus $\varphi(\bar{A}) \not \subset \bar{A}$. However one can easily check that

$$
\begin{aligned}
\Delta \varphi= & 4\left(\delta^{3}-\frac{3}{t} \delta^{2}+\frac{3}{t^{2}} \delta\right) \cup\left(t^{2} \delta\right)+6\left(t \delta^{2}-\delta\right) \cup\left(t \delta^{2}-\delta\right) \\
& +\left(t^{2} \delta\right) \cup\left(\delta^{3}-\frac{3}{t} \delta^{2}+\frac{3}{t^{2}} \delta\right) .
\end{aligned}
$$

Thus

$$
\Delta \varphi \in \operatorname{Der}_{Q}^{1}(A) \cup \operatorname{Der}_{Q}^{3}(A)+\operatorname{Der}_{Q}^{2}(A) \cup \operatorname{Der}_{Q}^{2}(A)+\operatorname{Der}_{Q}^{3}(A) \cup \operatorname{Der}_{Q}^{1}(A),
$$

but $\varphi(\bar{A}) \not \subset \bar{A}$.

This example shows that we really need the stronger statement $\varphi \in \operatorname{Der}_{k}^{n}(A)_{0}$ in order to conclude the $\varphi(\bar{A}) \subset \bar{A}$.

Finally, we note that the methods used in Theorems 1 and 2 give a new proof of A. Seidenberg's original theorem for finitely generated domains:

Theorem (A. Seidenberg). Let $A=k\left[x_{1}, \cdots, x_{g}\right]$ be a finitely generated integal domain over a field $k$ of characteristic zero. Let $\bar{A}$ denote the integral closure of $A$ in its quotient field $Q$. Let $\delta \in \operatorname{Der}_{k}^{1}(A)$. Then $\delta(\bar{A}) \subset \bar{A}$.

Proof. Using the same notation as in Theorem 1, we see that it suffices to prove $\delta(\bar{R}) \subset \bar{R}$. Write $\delta=a_{0} \delta_{0}+\cdots+a_{r-1} \delta_{r-1}$ with the $a_{i} \in Q$. Since $\delta\left(\alpha_{i}\right) \in R$, we see $a_{1}, \cdots, a_{r-1} \in R$. As before, it remains to argue that $v_{i}\left(a_{0}\right) \geqq 0$ for all $i=1, \cdots, t$. So fix an $i=$ $1, \cdots, t$ and assume $v_{i}\left(a_{0}\right)<0$. Pick $x \in R$ such that $N=v_{i}(x)$ is the minimum positive value of any element of $R$. Then $v_{i}(\delta(x))=v_{i}\left(a_{0}\right)+$ $N-1$. Since $\delta(x) \in R$, we conclude that $v_{i}\left(a_{0}\right)=1-N$. By an argument similar to that in Lemma 1 , we can find an element $y \in$ $R$ such that $M=v_{i}(y)$ is the minimum positive value of anything in $R$ which is not a multiple of $N$. Then $v_{i}(\delta(y))=M-N$ which is impossible.

\section{REFERENCES}

1. W. C. Brown, Higher derivations on finitely generated integral domains II, Proc. Amer. Math. Soc., 51 (1975), 8-14.

2. A. Grothendieck, Elements de Geometrie Algebreque IV, pt. 4, Pub. Math. de L'IHES \#32 Paris, 1967.

3. Y. Nakai, High order derivations I, Osaka J. Math., 7 (1970), 1-27. 
4. A. Seidenberg, Derivations and integral closure, Pacific J. Math., 16 (1966), 167173.

5. - Differential ideals in rings of finitely generated type, Amer. J. Math., 89 (1967), 22-42.

6. O. Zariski and P. Samuel, Commutative Algebra II, University Series in Higher Math. Van Nostrand, Princeton, N. J. 1958, MR 19 \#833.

Received March 25, 1975.

Purdue University

AND

Michigan State University 


\title{
PACIFIC JOURNAL OF MATHEMATICS
}

\author{
EDITORS
}

\author{
RICHARD ARENS (Managing Editor) \\ University of California \\ Los Angeles, California 90024
}

\author{
R. A. Beaumont \\ University of Washington \\ Seattle, Washington 98105
}

J. DugundjI

Department of Mathematics

University of Southern California

Los Angeles, California 90007

D. Gilbarg and J. Milgram

Stanford University

Stanford, California 94305

\section{ASSOCIATE EDITORS}
E. F. BECKENBACH
B. H. NEUMANN
F. WOLF
K. Yoshida

\section{SUPPORTING INSTITUTIONS}

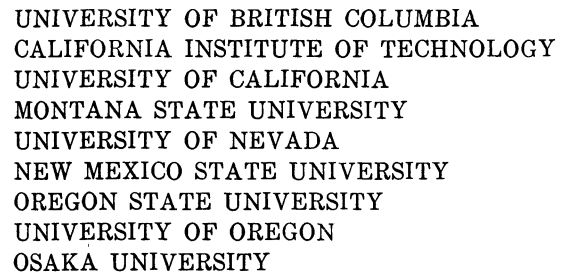

UNIVERSITY OF BRITISH COLUMBIA CALIFORNIA INSTITUTE OF TECHNOLOGY UNIVERSITY OF CALIFORNIA MONTANA STATE UNIVERSITY UNIVERSITY OF NEVADA NEW MEXICO STATE UNIVERSITY OREGON STATE UNIVERSITY UNIVERSITY OF OREGON OSAKA UNIVERSITY

\author{
UNIVERSITY OF SOUTHERN CALIFORNIA \\ STANFORD UNIVERSITY \\ UNIVERSITY OF HAWAII \\ UNIVERSITY OF TOKYO \\ UNIVERSITY OF UTAH \\ WASHINGTON STATE UNIVERSITY \\ UNIVERSITY OF WASHINGTON

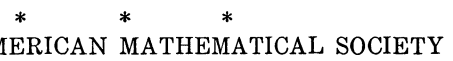

The Supporting Institutions listed above contribute to the cost of publication of this Journal, but they are not owners or publishers and have no responsibility for its content or policies.

Mathematical papers intended for publication in the Pacific Journal of Mathematics should be in typed form or offset-reproduced, (not dittoed), double spaced with large margins. Please do not use built up fractions in the text of your manuscript. You may however, use them in the displayed equations. Underline Greek letters in red, German in green, and script in blue. The first paragraph or two must be capable of being used separately as a synopsis of the entire paper. Items of the bibliography should not be cited there unless absolutely necessary, in which case they must be identified by author and Journal, rather than by item number. Manuscripts, in triplicate, may be sent to any one of the editors. Please classify according to the scheme of Math. Reviews, Index to Vol. 39. All other communications should be addressed to the managing editor, or Elaine Barth, University of California, Los Angeles, California, 90024.

The Pacific Journal of Mathematics expects the author's institution to pay page charges, and reserves the right to delay publication for nonpayment of charges in case of financial emergency.

100 reprints are provided free for each article, only if page charges have been substantially paid. Additional copies may be obtained at cost in multiples of 50 .

The Pacific Journal of Mathematics is issued monthly as of January 1966. Regular subscription rate: $\$ 72.00$ a year (6 Vols., 12 issues). Special rate: $\$ 36.00$ a year to individual members of supporting institutions.

Subscriptions, orders for back numbers, and changes of address should be sent to Pacific Journal of Mathematics, 103 Highland Boulevard, Berkeley, California, 94708.

PUBLISHED BY PACIFIC JOURNAL OF MATHEMATICS, A NON-PROFIT CORPORATION

Printed at Kokusai Bunken Insatsusha (International Academic Printing Co., Ltd.), 8-8, 3-chome, Takadanobaba, Shinjuku-ku, Tokyo 160, Japan.

Copyright (C) 1975 by Pacific Journal of Mathematics Manufactured and first issued in Japan 


\section{Pacific Journal of Mathematics}

\section{Vol. 63, No. 2 \\ April, 1976}

Joseph Anthony Ball and Arthur R. Lubin, On a class of contractive perturbations

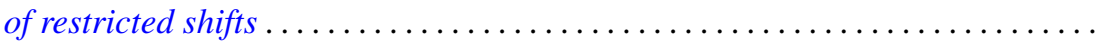

Joseph Becker and William C. Brown, On extending higher derivations generated

by cup products to the integral closure .......................

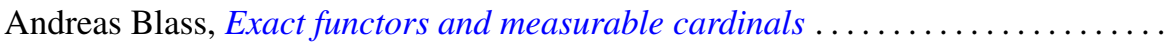

Joseph Eugene Collison, A variance property for arithmetic functions . . . . . . . . . .

Craig McCormack Cordes, Quadratic forms over nonformally real fields with a

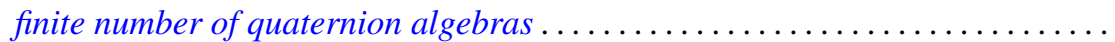

Freddy Delbaen, Weakly compact sets in $H^{1} \ldots \ldots \ldots \ldots \ldots \ldots \ldots \ldots \ldots$

G. D. Dikshit, Absolute Nörlund summability factors for Fourier series ..........

Edward Richard Fadell, Nielsen numbers as a homotopy type invariant. . ........

Josip Globevnik, Analytic extensions of vector-valued functions . . . . . . . . . . . .

Robert Gold, Genera in normal extensions . . . . . . . . . . . . . . . . . . . 389

Solomon Wolf Golomb, Formulas for the next prime

Robert L. Griess, Jr., The splitting of extensions of $S L(3,3)$ by the vector space

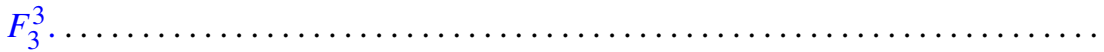

Thomas Alan Keagy, Matrix transformations and absolute summability .........

Kazuo Kishi, Analytic maps of the open unit disk onto a Gleason part.

Kwangil Koh, Jiang Luh and Mohan S. Putcha, On the associativity and commutativity of algebras over commutative rings . ..... . .

James C. Lillo, Asymptotic behavior of solutions of retarded differential difference equations.

John Alan MacBain, Local and global bifurcation from normal eigenvalues ..

Anna Maria Mantero, Sets of uniqueness and multiplicity for $L^{p}$

J. F. McClendon, Embedding metric families

L. Robbiano and Giuseppe Valla, Primary powers of a prime ideal .

Wolfgang Ruess, Generalized inductive limit topologies and barrelledness

properties.

Judith D. Sally, Bounds for numbers of generators of Cohen-Macaulay ideals

Helga Schirmer, Mappings of polyhedra with prescribed fixed points and fixed point indices.

Cho Wei Sit, Quotients of complete multipartite graphs

S. Sznajder and Zbigniew Zielezny, Solvability of convolution equations in $\mathscr{K}_{p}^{\prime}$,

$p>1$.

Mitchell Herbert Taibleson, The existence of natural field structures for finite

dimensional vector spaces over local fields

William Yslas Vélez, A characterization of completely regular fields

P. S. Venkatesan, On right unipotent semigroups ..............

Kenneth S. Williams, A rational octic reciprocity law ............

Robert Ross Wilson, Lattice orderings on the real field .......... 